\title{
Unification and Mathematical Explanation in Science
}

\author{
Sam Baron*
}

\begin{abstract}
Mathematics clearly plays an important role in scientific explanation. Debate continues, however, over the kind of role that mathematics plays. I argue that if pure mathematical explananda and physical explananda are unified under a common explanation within science, then we have good reason to believe that mathematics is explanatory in its own right. The argument motivates the search for a new kind of scientific case study, a case in which pure mathematical facts and physical facts are explanatorily unified. I argue that it is possible for there to be such cases, and provide some toy examples to demonstrate this. I then identify a potential source of scientific case studies as a guide for future work.
\end{abstract}

\section{INTRODUCTION}

There has been a recent surge of interest in mathematical explanations in science. ${ }^{1}$ While it is generally agreed that mathematics plays some role in scientific explanation, there is substantial disagreement over what kind of role mathematics plays. According to some, mathematics is explanatory in its own right. There are physical phenomena that obtain for mathematical reasons. ${ }^{2}$ According to others, mathematics itself is not explanatory. Rather, mathematics merely represents underlying physical facts, and it is these facts that are doing the explanatory work. ${ }^{3}$

In this paper, I present an argument for the view that mathematics is explanatory. The argument can be viewed as the extension of certain core ideas found within Kitcher's (1981; 1984) unificationist approach to explanation. However, the argument does not rely on endorsing Kitcher's specific unificationist theory. Rather, it draws on an idea, at times suggested by Kitcher (1984, pp. 218-220), that explanatory unification is an important aspect of both scientific explanation and explanation within pure mathematics. I take this idea a step further, and suggest that physical and mathematical phenomena might be explanatorily unified and that this can lead us to believe that mathematics is explanatory in its own right. ${ }^{4}$ The argument that I present for the explanatory power of mathematics thus

\footnotetext{
*Dianoia Institute of Philosophy, Australian Catholic University. Email: samuel.baron@acu.edu.au

${ }^{1}$ For discussion, see Andersen (2016); Baker (2005, 2009, 2017); Bangu (2020); Baron (2020); Baron et al. (2017); Chirimuuta (2018); Colyvan (2001, 2010); Jansson and Saatsi (2019); Knowles and Saatsi (2019); Knowles (forthcoming); Lange (2016); Leng (2010); Lyon (2012); Pincock (2015, 2007); Povich (2019); Saatsi $(2017,2016)$.

${ }^{2}$ This view is defended by Baker (2005); Baron (2020); Colyvan (2010); Lange (2016); Potochnik (2007); Pincock (2007) among others.

${ }^{3}$ See, for instance, Bueno (2012); Liggins (2012); Leng (2010); Melia (2000); Saatsi (2011); Yablo (2012).

${ }^{4}$ The approach is also reminiscent of Steiner's (1978b) account, on which mathematics plays a genuine explanatory role in a scientific explanation if, when we strip out all of the physical detail of the explanation, an explanation of a pure mathematical fact is left over. See Baker (2012) and Lyon (2012) for recent discussion.
} 
motivates the search for a particular kind of scientific case study: a case in which pure mathematical facts and physical facts are explanatorily unified. Unfortunately, no such case has yet been identified within the philosophical literature. I argue, however, that it is possible for there to be such cases, and provide some toy examples to demonstrate this. I then identify a potential sources of realistic cases as a guide for future work.

\section{The Argument}

Following Saatsi (2016), let us differentiate between a 'thick' and a 'thin' explanatory role for mathematics. This distinction can be captured, roughly, as follows: ${ }^{5}$

\section{Thick}

Mathemetics plays a thick explanatory role when it is part of the reason why something physical obtains; it is responsible for some physical phenomenon.

\section{Thin}

Mathematics plays a thin explanatory role when it merely represents the reason why something physical obtains; it is not responsible for some physical phenomenon.

Underlying the distinction between thick and thin explanatory roles is a corresponding distinction between two uses of the word 'explanation'. First, an explanation is a representation: usually a model, theory, argument or set of sentences, but not only. A claim stating the explanandum is generally an output of the model, a conclusion of the argument, or an implication of the theory or set of sentences. Call this an $R$-explanation. Science is in the business of producing and offering explanations in this sense.

Second, an explanation is a part of the world. The world contains various features, and sometimes one feature is responsible for another. When one feature of the world is responsible for another, we say that the first is the explanation for the second: it is the reason why the second occurred. Call this an $O$-explanation. There are different types of responsibility that might underwrite an $O$-explanation. It may be, for instance, that $P$ is responsible for $Q$ only if $P$ causes $Q$, or only if $P$ necessitates $Q$ via some modality or other, or only if $Q$ is grounded in $P$. It may be that all of these types of responsibility are present in the world, and so there are different kinds of $O$-explanations: causal, modal, grounding and so on.

When an explanation in the first sense - an $R$-explanation - is accurate it represents an explanation in the second sense - an $O$-explanation - and thus manages to capture some explanatory relation between worldly features. When mathematics features in a scientific $R$-explanation of some physical fact, but not in any $O$-explanation of that fact, then mathematics is playing a thin role. When mathematics features in an $O$-explanation of some physical fact, then mathematics is playing a thick explanatory role.

\footnotetext{
${ }^{5}$ Note that the distinction between the thick and thin explanatory roles is not supposed to be the same as the distinction between the ontic and epistemic accounts of explanation. Saatsi's idea is that even if we assume that explanations are generally ontic and that the explanatory power of our scientific representations comes from representing objective relations of explanatory relevance in the world, we can still draw a distinction between two roles for mathematics. Mathematics might appear in our scientific representations merely as a way of representing physical facts that are doing the explanatory work; or mathematics might itself be doing the explanatory work. The thick/thin distinction should thus be read as internal to the ontic account of explanation.
} 
Clearly, mathematics plays at least a thin explanatory role in science. Scientific $R$ explanations are shot-through with mathematical content. The question, though, is whether mathematics is ever playing a thick role. As noted above, for some philosophers, the answer is 'yes'. The case for the affirmative is based on indispensability considerations. If mathematics is indispensable to an $R$-explanation-if removing the mathematics would weaken the explanatory power of the representation to accurately represent some $O$ explanation - then mathematical facts must be part of the $O$-explanation that the relevant scientific theory or model represents.

Saatsi (2016) argues that any inference along these lines faces a problem. The difficulty lies with the representational capacity of mathematics. Mathematics is capable of representing various physical aspects of the world. So when mathematics appears in a representation and imbues that representation with greater explanatory power, it might be because the representation is more accurately representing the mathematical reasons why something occurs. Equally, however, it could be that the mathematics is merely representing some underlying physical fact, allowing that fact to play a role in the representation. The boost to explanatory power yielded by the mathematics could be entirely due to the fact that the inclusion of mathematics enables the representation to more accurately capture the physical reasons why something happens.

Saatsi's concern, then, is that being indispensable to an $R$-explanation underdetermines what kind of explanatory role mathematics plays. To address the concern, we need a way to determine when a model or theory implies that mathematics is part of the reason why some physical fact obtains. If we could simply peruse the worldly facts and directly observe what explains what, then perhaps it would be easy to determine whether mathematics ever plays a thick role. The trouble, however, is that our best access to what explains what often goes via the representations that science provides, and it does so exclusively when we cannot directly observe whatever is supposed to be doing the explanatory work (as is surely the case with mathematics). It is thus difficult to see how we could ever come to know that mathematics is playing a 'thick' versus a 'thin' explanatory role. Science itself seems to be screening off our epistemic access to $O$-explanations in the case of mathematics and we cannot easily peer behind the curtain.

In what remains of this section, I present an argument for the view that mathematics is playing a thick explanatory role. The argument relies on unification. I'm not the first to appeal to unification as a way to establish that mathematics is playing a thick role. ${ }^{6}$ But, so far as I can tell, all previous appeals fall prey to the under-determination worry that Saatsi raises. The argument presented here avoids Saatsi's worry. The basic idea is this: if physical phenomena are unified with pure mathematical phenomena within some $R$-explanation in our best science, then we should believe that mathematics is playing a thick explanatory role in explaining the relevant physical phenomena.

In order to present the argument, I need to say a bit more about the kind of explanatory unification at issue. I will differentiate between two kinds of unification: vertical unification, which is a feature of scientific representations; and horizontal unification, which is a feature of the world. Vertical unification is the unification of two or more explananda under a common $R$-explanation, such as a specific model or theory. The degree of vertical unification for an explanation is simply a measure of the number of distinct explananda that a model outputs or that a theory implies. Horizontal unification, by contrast, relates

\footnotetext{
${ }^{6}$ Similar appeals have been made by Baker (2017) and by Colyvan $(2001,2002)$.
} 
to the reasons why things occur and so it is a feature of $O$-explanations. Very roughly, explananda sometimes have the same reasons for being, and when they do they are horizontally unified.

If two explananda are vertically unified within an $R$-explanation that is part of our best science, then we should believe that those explananda are horizontally unified as well. The motivation for this claim is based on a commitment to scientific realism. ${ }^{7}$ Assuming a realist outlook on science, we should accept that our best scientific theories are true. ${ }^{8}$ When two explananda are unified under a single $R$-explanation within a theory $T$, then that theory represents the two explananda at issue as having the same $O$-explanation. If those two explananda in fact have different $O$-explanations, then the theory gets the $O$ explanation for at least one of those explananda wrong. A true theory, however, should not make this kind of mistake, and so we should expect vertical unification in a true theory to accurately represent horizontal unification. ${ }^{9}$

Now, suppose we discover that there is a scientific $R$-explanation that vertically unifies a physical explanandum $P$ and a mathematical explanandum $M$. Then it follows that those explananda are horizontally unified and thus share a single $O$-explanation. From there, it is possible to formulate the following argument:

(1) Physical fact $P$ and mathematical fact $M$ are vertically unified within our best science.

(2) If two facts $M$ and $P$ are vertically unified within our best science, then the $O$-explanations for $M$ and $P$ overlap.

(3) The $O$-explanation for every mathematical fact is mathematical.

(4) If (1), (2) and (3) then the overlap between the $O$-explanations for $M$ and $P$ is mathematical in nature.

(5) If the overlap between the $O$-explanations for $M$ and $P$ is mathematical in nature, then there is at least one physical fact $P$ that obtains, in part, for mathematical reasons.

Therefore,

(6) There is at least one physical fact $P$ that obtains, in part, for mathematical reasons.

\footnotetext{
${ }^{7}$ I am grateful to a referee for the following way of stating the motivation.

${ }^{8}$ Why believe that our best scientific theories are true? This is due, primarily, to the so-called 'nomiracles' argument. This argument proceeds, roughly as follows: our current best scientific theories are extremely well-confirmed and enjoy incredible predictive success. The best explanation of this is that they are true. So we should believe that they are true. The literature on this argument is extensive. For an excellent overview and defense of the no-miracles argument, see Psillos (1999). For a compelling case against the no-miracles argument see Frost-Arnold (2010). For a critical discussion of the use of realism to support indispensability arguments, see Saatsi (2007).

${ }^{9}$ Some scientific realists argue only for the approximate truth of our best scientific theories. In the present context, the notion of approximate truth is potentially troubling. For it is compatible with the approximate truth of our best theories that vertical unification does not always imply horizontal unification, so long as our theories get it right enough of the time. Approximate truth poses a rather general problem for indispensabilitybased arguments that focus on explanation. For if our best theories can make mistakes, then one of those mistakes can just be the mistake of incorrectly implying that mathematics is playing a thick explanatory role. I think this is a genuine concern but, due to its generality, not one that I have the space to deal with here.
} 
Premise (1) assumes that there are scientific explanations that unify mathematical and physical explananda, an assumption to be discussed in $\S 3-4$. Premise (2) follows from the proposed connection between vertical and horizontal unification. If $P$ and $M$ are vertically unified within our best science, then the $O$-explanations for $P$ and $M$ are horizontally unified and thus overlap. Premise (3) will be defended in a moment. Given Premise (3), the only reasons why $M$ obtains are mathematical in nature. Thus if $P$ and $M$ share some common reason for being - if their $O$-explanations overlap - then it has to be mathematical. There is no other reason that the two could hold in common, certainly no physical reason. This gives us Premise (4). Given that the only reasons for being that the $O$-explanations for $P$ and $M$ can share in common are mathematical ones, it follows that $P$ obtains for partly mathematical reasons.

Although the argument does not explicitly appeal to indispensability, indispensability is playing a role nonetheless. The $R$-explanations at issue must be part of our best science. Being a part of our best science usually implies indispensability and so it is only indispensable $R$-explanations that we have reason to believe accurately represent $O$-explanations. It is the indispensability of the $R$-explanations that therefore gives us reason to believe in the presence of $O$-explanations of the mathematical and physical facts at issue.

Furthermore, vertical unification is indispensable to our best theories only if it tracks horizontal unification in the proposed manner. So it is the indispensability of vertically unifying $P$ and $M$ in the above argument that makes the difference. But exactly how the unification is achieved is not important. The unification would, presumably, be achieved by using mathematics to formulate a unifying $R$-explanation. But the unification could, conceivably, be achieved by formulating an $R$-explanation that makes no explicit reference to mathematics. So long as at least one explanandum is mathematical and the other is physical, that's all that really matters. That being said, the argument still implies that mathematics is indispensable to scientific explanation, in so far as it is needed for $O$-explanations. If, as Field (1980) argued, mathematics is dispensable to scientific explanations including $O$-explanations, then the present argument fails.

Why accept Premise (3) of the argument? In order to accept Premise (3) one must be willing to accept that there are, or at least can be, mathematical explanations of mathematical facts. If one believes that there are no such explanations, then one will have reason to doubt Premise (3) and, indeed, the set-up of the argument. For present purposes, however, I will simply assume that there are mathematical explanations of mathematical facts, and note that this is controversial (more on this in a moment). ${ }^{10}$ For now, the main aspect of Premise (3) to defend is the idea that mathematical facts can't hold for physical reasons. Since if mathematical facts can hold for physical reasons, then the overlap between the $O$-explanations for $P$ and $M$ might be physical after all, in which case there is an ordinary physical reason for $P$.

I am willing to concede that some $R$-explanations of mathematical facts may feature aspects that appear to be physical and not mathematical in nature (see Skow 2015). It can sometimes be helpful to frame mathematical facts in physical terms in order to explain those facts. So, for instance, it can be useful for understanding various geometric facts that we frame our explanations of those facts in spatial or temporal terms. But even if $R-$ explanations feature aspects that appear to be physical, it does not follow, without further argument, that physical facts feature in any $O$-explanation for a mathematical fact. The

\footnotetext{
${ }^{10}$ See Zelcer (2013) for scepticism about mathematical explanation.
} 
reason for this is that it is possible to represent purely mathematical facts using physical language. Accordingly, when physical language appears in an $R$-explanation for a mathematical fact, it could be the case that the physical language represents some physical fact. Equally, however, it could be that the physical language represents some mathematical fact.

This is a version of Saatsi's point about the use of mathematics in science. As already discussed, the use of mathematics in $R$-explanations of physical facts under-determines the role that mathematics plays in explanation. In particular, it does not settle whether the reason why a physical fact obtains is mathematical. By the same token, even if an $R$-explanation for a mathematical fact features physical features, that under-determines whether some physical fact is the reason why a mathematical fact obtains.

Now, one might worry that I am guilty of holding a double standard. For it seems I am claiming that the use of mathematical elements in an $R$-explanation for a physical fact implies that mathematical facts explain physical facts, while simultaneously denying that the use of physical elements in an $R$-explanation for a mathematical fact implies that physical facts explain mathematical ones. In fact, however, I am conceding in both cases that just because an $R$-explanation contains certain features - physical or mathematical - we cannot thereby conclude anything about what really explains what. Some further argument is required to show that mathematical facts explain physical ones or vice versa. Above, I have offered such an argument for the case of mathematical explanations of physical facts. The point is that an argument in the case of physical explanations of mathematical facts is currently lacking.

If such an argument could be provided then that would cause trouble for my project. I am sceptical, however, that such an argument can be provided. That's because there are reasons to deny that mathematical facts could ever be explained by physical facts. This is so for two reasons. ${ }^{11}$ First, it is plausible that the reason why a mathematical fact obtains is always the same reason. Which is to say we don't have a situation where, in one world, there is one reason why a mathematical fact obtains, and in another world there is a different reason why the same fact obtains. This has something to do with the necessity of mathematical facts, which is connected to their nature as mathematical facts. The necessity of mathematical facts seems to demand a modally uniform explanation for why those facts hold. If any mathematical fact ever has a physical explanation, however, then because no physical fact holds in every world, there must be a world in which the reason why the mathematical fact in question obtains is not the physical fact at issue. This undermines the claim that the mathematical fact holds for the same reason in every world.

Second, there is a modal mismatch between mathematical and physical facts that seems to make physical facts unfit to explain mathematical ones. Suppose that a physical fact is the reason why some mathematical fact obtains. It is plausible to suppose, in general, that if $x$ is the reason for $y$, then the loss of $x$ would or at least might bring about the loss of $y$. Because physical facts are contingent, there are situations in which the physical facts go missing. If this forces the mathematical facts to go missing as well, then this seems to inject an unwanted element of contingency into the mathematical facts. Conversely, if

\footnotetext{
${ }^{11}$ Skow (2015) considers similar reasons to the two cited here. To a certain extent he concedes the force of the first reason, though he still maintains that physical facts can be used to explain mathematical facts in at least one case (involving Pythagoras's theorem). Though I can't argue the point here, the example he uses strikes me as one in which physical facts appear in the $R$-explanation for a mathematical fact, but are not part of the reason why the fact holds.
} 
mathematical facts are necessary, then it is difficult to see how they could be responsive to variability in the physical facts in the manner that explanation seems to require.

Note that the two reasons just adduced for thinking that mathematical facts cannot be explained by physical facts don't work in the opposite direction: as reasons for thinking that physical facts cannot be explained by mathematical facts. On the one hand, there does not seem to be the same presumption that physical facts should always hold for the same reason. So the first point made above doesn't apply in the case of mathematical explanations of physical facts. On the other hand, the modal mismatch between mathematical and physical facts does not obviously preclude the former from explaining the latter. The modal mismatch is only a problem when the explanandum is necessary and the explanans is contingent. For then there have to be possible situations in which the explanandum is present, but the explanans is absent. But when the explanandum is necessary and the explanans is contingent — as when a mathematical fact explains a physical one — such a situation never arises.

Note also that by appealing to the necessity of mathematics in the manner above, I am not espousing a view according to which mathematics is explained by its necessity. The idea, rather, is that because mathematical facts are necessary, they are unfit to be explained by physical facts. It is thus a necessary condition on the explanation of a mathematical fact that the explanans holds of mathematical necessity. But it is by no means sufficient for explaining a mathematical fact, that there is some other mathematical fact that holds with a similar degree of necessity.

One might remain worried about Premise (3): even if mathematical facts don't hold for physical reasons, it might still be the case that mathematical facts don't always hold for mathematical reasons, in which case Premise (3) might not be true. As I see it, there are two reasons to believe that mathematical facts hold for mathematical reasons. First, to ensure that a given mathematical fact holds for the same reason in every situation, the reason ought to be a mathematical fact. Since only then can we be assured that the two facts will 'line up' modally speaking, since the two facts will both hold of mathematical necessity.

Second, it is difficult to see what else might explain a mathematical fact. One might gesture toward metaphysical facts. But these facts seem to be of the wrong kind to explain mathematics and, at any rate, are not mathematically necessary (and so suffer a similar fate as physical facts). One might point toward logical facts, but it is not at all obvious that a sharp distinction can be maintained between logical and mathematical facts. Besides most mathematical facts don't hold for logical reasons (if any do) and so I don't really see this as a viable way to resist Premise (3). ${ }^{12}$

One might still be worried about Premise (3), but for a different reason. The worry, in brief, is that Premise (3) makes the argument question-begging. ${ }^{13}$ In order to accept that premise, one must assume that there are mathematical $O$-explanations for mathematical

\footnotetext{
${ }^{12}$ If one remains worried, however, then there is a weaker version of Premise (3) that will do just as well. Namely: the $O$-explanation for $M$ is mathematical. We can thus restrict the argument to those mathematical facts that do not hold for some purely logical reason. This, I take it, is something we may be able to determine using the tools of mathematics and logic (assuming there's a clean split between the two). What if there are 'brute' mathematical facts? Such cases don't pose a problem for the argument. Premise (3) is a universal claim and so it can be read in a conditional sense: if a mathematical fact has an $O$-explanation, then it's $O$ explanation is mathematical. The fact that a mathematical fact is the output of an $R$-explanation is a reason to believe it has an $O$-explanation and thus that the antecedent is satisfied.

${ }^{13}$ I am grateful to a referee for raising this objection.
} 
facts. However, the existence of $O$-explanations involving mathematics is precisely what we are trying to establish, since if this can be established then mathematics is playing a thick explanatory role after all.

Premise (3) should, however, be read in a conditional sense: if there are any $O$ explanations for mathematical facts, then those $O$-explanations should be mathematical (for the reasons just discussed). The fact that there are $O$-explanations for mathematical facts is not presupposed by Premise (3) but, rather, gets established by Premise (1). The fact (if it is one) that there are $R$-explanations in science that output mathematical explananda implies that those mathematical explananda have $O$-explanations (assuming that all true $R$-explanations in science represent $O$-explanations and our scientific $R$-explanations are true). Thus, the existence of $O$-explanations for mathematics issues from whatever basis we have for believing scientific realism, plus the presence of certain kinds of explanations in science.

But, one might respond, if the argument establishes that there are $O$-explanations for mathematical facts and what we are trying to show is that mathematics plays a thick explanatory role, then anything else that the argument does is redundant. There is no need to also show that mathematics features in the $O$-explanations for physical facts.

At worst, this problem represents an embarrassment of riches. What the argument does is produce two reasons to believe that mathematics is playing a thick explanatory role: it plays this role both with respect to mathematical facts and with respect to physical facts. Ultimately, however, it is important to show both things. That is because one can always adopt a conservative approach to ontological commitment, according to which only $O$-explanations of physical phenomena should be deemed ontologically committing. Assuming this conservative approach, the existence of $O$-explanations for mathematical facts is not sufficient to warrant realism toward mathematical objects. If the aim is, ultimately, to make a case for realism, then the full argument stated above is required.

Is there another reason to suppose that the argument is question-begging? I have claimed that the existence of $O$-explanations for mathematical facts gets established by realism about our scientific theories, specifically those that include $R$-explanations that unify physical and mathematical explananda. But, one might argue, surely we can only have a reason to believe a scientific theory that features $R$-explanations of this kind if we already have evidence that is specifically about the existence of $O$-explanations featuring mathematics. But precisely what we are seeking is evidence of this kind; evidence that can provide a reason to believe that there are such $O$-explanations in the first place.

However, the evidence in favour of a given scientific theory-evidence that leads us to believe that a scientific theory is well-confirmed and thus true - need not be evidence that is specifically about every part of that theory, and thus about every explanation that features in the theory. It is, after all, possible to discover new explanations of novel phenomena simply in virtue of confirming a theory and then fully exploring its implications. That is how I see the situation with respect to $O$-explanations for mathematical facts. We don't need evidence in favour of such explanations beyond whatever evidence we have for a given scientific theory. The reason to believe in the existence of these explanations is based on the broader confirmation of the theory in which they are embedded.

The reasoning here relies on explanation holism: we should believe all of the explanations that are embedded in a confirmed theory. Explanation holism is similar to confirmational holism. Moreover, explanation holism is doing similar work in the present context that confirmational holism is often made to do in indispensability arguments. It is because 
confirmation is holistic in some sense, that $O$-explanations for mathematics are indirectly established via the confirmation of a scientific theory. Unlike confirmational holism, however, explanation holism does not require a commitment to every entity posited by a confirmed theory. It therefore does not face the same problems as confirmational holism that plagued early versions of the indispensability argument.

Explanation holism helps me to deal with a further charge of question-begging. One might worry that the argument I have presented requires not only $O$-explanations for mathematical facts, it requires the mathematical facts themselves. For, one might argue, scientific explanation is factive in this sense: $A$ can only be explained if $A$ is a fact. Thus, as soon as I assume that there are $R$-explanations within science that unify both physical and mathematical explananda, I have already assumed that there are mathematical facts to be explained. If there are mathematical facts then, one might think, it follows that mathematical objects exist. But the style of argument I am trying to put forward is supposed to pave a way to realism about mathematics. So it shouldn't assume that view.

However, I am not flatly assuming that there are mathematical facts to be explained. The existence of these facts, like the $O$-explanations for them, gets established by the fact that there are $R$-explanations in our best science that output mathematics. For if we have reason to believe that these $R$-explanations are true, and explanation is factive in the above sense, then it follows that the mathematical facts at issue are true. Thus, in the case of mathematical facts, both the explanans and the explanandum are established together via realism about our best theories. This is unusual, but not incoherent (a similar process of bootstrapping the explanandum via the explanans is discussed by Baker (2009, pp. 620622)). As before, the evidence in favour of the theory need not be evidence that directly supports the mathematical facts in question. The evidence can support the theory in a general sense, with the mathematical facts and their explanations caught up in the same act of confirmation.

Once again, a charge of redundancy seems to apply: if my argument establishes that there are mathematical facts, then the day is won for the mathematical realist and there is no need to go any further. As before, however, one may adopt a more conservative picture of ontological commitment, according to which we should be ontologically committed to something only if it plays a role in $O$-explanations for physical phenomena. In this case, the rest of the argument is needed.

\section{TOY CASES}

The argument presented in the previous section provides a template for establishing that mathematics plays a genuine explanatory role in science. The template must be filled in by particular cases: examples in which mathematical and physical facts are explanatorily unified. Have any such cases been identified already? At first glance, a range of cases identified by Steiner seem to be of the right kind. Steiner appeals to explanations within mathematics as a basis for identifying cases of mathematical explanation within science. His account thus involves a blending of pure mathematical explanation and physical explanation.

On Steiner's (1978b) view, mathematics is playing a genuine explanatory role in a scientific $R$-explanation of some physical phenomenon when, were we to remove the physical details of the $R$-explanation we would be left with an explanatory proof of a mathematical 
fact. For Steiner (1978a, p. 142), a mathematical proof of $\mathrm{P}$ is explanatory when, roughly, the proof holds in virtue of some characterising property of $\mathrm{P}$ (which, for present purposes, we can understand as an essential property) such that it is clear how the demonstration of $P$ depends on that property.

Steiner's account thus relies on the idea that explanatory proofs are embedded in $R$ explanations within our best science. Steiner's primary example is the explanation of why it is that the displacement of a rigid body around a fixed point can always be understood as displacement around some axis. This explanation, he argues, makes use of a result in pure mathematics involving complex numbers (see Steiner (1978b) for the details). We then add some physical assumptions to the picture, such as the assumption of a physical, Euclidean space, and a further physical explanation is yielded.

Suppose that Steiner is correct and mathematical explanations of mathematical facts are sometimes embedded inside $R$-explanations of physical facts. Such cases seem to unify explanations of mathematical and physical facts: a single $R$-explanation explains both why a mathematical fact obtains, in virtue of the embedded proof, and why some physical fact obtains, in virtue of the added physical assumptions that enable the mathematics to be applied to a physical system.

Unfortunately, these cases are not of the right kind to support the argument outlined in the previous section. $R$-explanations that fit Steiner's picture certainly entail mathematical explanations of mathematical facts in virtue of the embedded mathematical proof. But this is not the same thing as unifying a mathematical and a physical fact under a common explanation. Indeed, the mathematical and physical facts have distinct explanations. The mathematical explanation makes no use of the physics that is needed to explain the physical explanation, and the physical explanation makes use of more than the mathematics alone.

A more promising case is offered by Colyvan (2001, pp. 81-83). According to Colyvan, complex numbers play a unifying role in pure mathematics. In particular, they are needed to unify trigonometric and exponential functions. This can be achieved by introducing a complex number $i=\sqrt{-1}$ and defining a complex variable $z$ over real numbers $x$ and $y$, such that $z=x+y i$. The exponential function can then be stated as a function of $i$ via Euler's formula as follows:

$$
e^{i \theta}=\cos \theta+i \sin \theta \quad \theta \in \mathbb{R}
$$

Which can then be used to state the trigonometric function for $z$ :

$$
\sin z=\frac{e^{i z}-e^{-i z}}{2 i} \text { and } \cos z=\frac{e^{i z}+e^{-i z}}{2}
$$

Colyvan (2001, pp. 81-83) goes on to claim that the unification of the trigonometric and exponential functions "flows through" into explanations of physical phenomena, and thus that the unification provided by the complex numbers is a "fine example of the unity a mathematical theory may bring not only to other mathematical theories but also to scientific theory as a whole" (Colyvan, 2001, p. 83).

The example that Colyvan offers of how the mathematical unification reaches into physics involves the use of second-order, linear differential equations of the following general form:

$$
y^{\prime \prime}+y^{\prime}+y=0
$$


Where $y$ is a real-valued function of a single real variable $x$. Equations of this kind are solved by solving their characteristic equation: $r^{2}+r+1=0$, which has two roots: $r=-\frac{1}{2} \pm i \frac{\sqrt{3}}{2}$. A general solution to the equation takes the form $y=c_{1} e^{r_{1} x}+c_{2} e^{r_{2} x}$ in which $c_{1}$ and $c_{2}$ are real constants and $r_{1}$ and $r_{2}$ are the two roots of the characteristic equation. The general solution admits both real and complex roots, and so there are roughly two ways to solve the characteristic equation. What Euler's formula allows us to do is unify the complex and real solutions, by enabling a reduction of one to the other (see Colyvan (2001, p. 83) for the details of the reduction with respect to $y^{\prime \prime}+y^{\prime}+y=0$ ).

Importantly, differential equations of the above form can require real or complex solutions, depending on the signature of the equation (e.g., $y^{\prime \prime}+y=0$ has a complex solution and $y^{\prime \prime}-y=0$ has a real solution). Without the unification of the exponential and trigonometric functions which effectively unites complex and real solutions, it would not be possible to unify the two methods of solving these equations within a single mathematical framework. As Colyvan notes, this may look merely like a kind of 'algorithmic' unification. We are, after all, unifying two distinct ways of solving the characteristic equation for $y^{\prime \prime}+y^{\prime}+y=0$. However, he maintains that this unification occurs only in virtue of a deep structural relationship between the two types of equation $y^{\prime \prime}+y=0$ and $y^{\prime \prime}-y=0$.

Colyvan goes on to claim that the mathematical unification of the real and complex methods of solving $y^{\prime \prime}+y=0$ and $y^{\prime \prime}-y=0$ also explains similarities between physical systems. Here's the general idea. Suppose we have two physical systems $S_{1}$ and $S_{2} . S_{1}$ is governed by a differential equation of the form $y^{\prime \prime}+y=0$ and $S_{2}$ is governed by an equation of the form $y^{\prime \prime}-y=0$. Suppose that the two systems display similar behaviour. One potential explanation for that similarity is that the solutions to the two characteristic equations governing those systems are mathematically unified in the sense described above. The deep structural similarities that Colyvan appeals to between the constraint equations $y^{\prime \prime}+y=0$ and $y^{\prime \prime}-y=0$ also explain the physical similarities between systems.

If Colyvan is right, then there may be cases in which the mathematical unification provided by the complex numbers is helping to explain both a physical fact and a mathematical fact. The unification in such a case would explain why the equations $y^{\prime \prime}+y=0$ and $y^{\prime \prime}-y=0$ share a common solution which, in turn, explains why physical systems described by those equations share common physical behaviour. This picture effectively exploits the transitivity of explanation to get an example of roughly the right kind. A mathematical fact $M$ explains another mathematical fact $M^{*}$, which in turn explains a physical fact $P$.

While initially promising, Colyvan's case cannot clearly support the argument offered in $\$ 2$ either. In Colyvan's example, the proposed $R$-explanation would be an explanation of the physical similarities between two physical systems. The similarities are explained in virtue of a particular case of mathematical unification: the fact that the methods of solving the constraint equations can be unified. While this fact about unification needs to appear in the $R$-explanation, the further mathematical fact that explains the unification-Euler's formula - isn't strictly required to explain the physical facts. So the $R$-explanation itself doesn't explain the unification of the solutions to $y^{\prime \prime}+y=0$ and $y^{\prime \prime}-y=0$, it merely uses it. If we did include Euler's formula we would have a case of embedding of the type identified by Steiner. The $R$-explanation would, at best, involve adding some physical facts to the explanation of why the real and complex solutions to $y^{\prime \prime}+y=0$ and $y^{\prime \prime}-y=0$ are unified.

In sum, then, neither Steiner's cases nor Colyvan's are examples in which physical and 
mathematical facts are explanatorily unified in the right way. Moreover, it is difficult to think of a better example. This might lead one to be rather pessimistic about the prospects for the argument outlined in $\S 2$. This scepticism can be met to a certain extent by considering some toy examples. Such examples show that it is at least possible for mathematical and physical facts to be explanatorily unified; there is no in principle reason why explanatory unification of this kind should be ruled out.

Toy examples of the right kind are in fact quite easy to produce. The easiest way to produce them is to consider a certain class of mathematical explanations identified by Lange (2016), explanations that he calls 'distinctively mathematical explanations'. These are explanations in which a certain physical fact is explained entirely in terms of one or more mathematical facts. These cases are already very close to pure mathematical explanations, in so far as they don't rely on any physical facts. As a result, such examples tend to have a pure mathematical analogue.

A number of toy cases can be produced by following a simple recipe. The recipe is just this: start with a distinctively mathematical explanation, identify a pure mathematical analogue (i.e., a pure mathematical case that uses the same mathematics) and then unify the two cases under a single explanation. To see the idea, I will briefly outline two examples.

The first example is based on one of Lange's (2016, p. 9) cases. Suppose that Sara has a piece of yarn and she is trying to unknot it without cutting the yarn. Unfortunately, the yarn contains a trefoil knot. Given this, it is impossible for Sara to untie the knot. Why?

The answer lies in knot theory. A knot in geometry is a line segment in three dimensions that has been wrapped around itself arbitrarily and then joined to form a loop. The most basic form of knot is a simple loop. This is the unknot. One knot $K_{1}$ is equivalent to another knot $K_{2}$ when there is an orientation-preserving homeomorphism between the two knots in three dimensions. The trefoil knot is not equivalent to the unknot. A physical knot in a piece of yarn in three dimensions can be untied without cutting the yarn only if the corresponding mathematical knot is equivalent to the unknot. It is therefore impossible for Sara to untie the knot in her yarn because she is dealing with a trefoil knot. We can formulate this case, as follows:

Yarn: Sara cannot untie the trefoil knot in her yarn because the trefoil knot is distinct to the unknot.

Sara's yarn trouble has a close mathematical analogue. Suppose that we have two knots in the purely mathematical sense-i.e., not physical knots in Sara's or anyone's yarn. One knot is the trefoil knot. We want to know whether the trefoil knot can be 'untied'. The mathematical equivalent of physically untying a knot is an algorithm by which one knot is converted into another via a sequence of transformations. The steps in this algorithm are known as Reidemeister moves. There is no sequence of Reidemeister moves that takes one from a trefoil knot to the unknot. Why? Well, there is such a set of moves only if there is an orientation-preserving homeomorphism between the two knots. Which is to say, only if the two knots are equivalent. This gives us the following:

Trefoil: There is no sequence of Reidemeister moves from the trefoil knot to the unknot because the trefoil knot is distinct to the unknot.

One might worry that the explanation is trivial: what it means for two knots to be equivalent is for there to be a set of Reidemeister moves that takes one to the other. But while 
there is a mutual entailment between the fact that two knots are equivalent and the fact that there is a sequence of Reidemeister moves from one knot to another, these are different facts and so the explanation is non-trivial. Equivalence between two knots is a function: a mapping from one knot to another that preserves (at least) topology. Reidemeister moves by contrast are not mappings, they are algorithms which involve the step-wise transformation of line segments.

Sara's yarn trouble and the pure knot theory case share a single explanans. Accordingly, the explanations can be combined into a single, unified explanation with both physical and mathematical explananda. The combined explanation can be stated as follows:

Yarn-Trefoil: The reason why Sara cannot untie the trefoil knot in her yarn and the reason why there is no sequence of Reidemeister moves from the trefoil knot to the unknot is the same, namely the trefoil knot is distinct to the unknot.

The second example is based on the well-known Bridges of Königsberg case introduced into the philosophical literature by Pincock (2007), which Lange also cites as a distinctively mathematical explanation. In Königsberg there were once seven bridges connecting a sequence of islands. It was impossible to cross all seven bridges by crossing each bridge exactly once. Why? The answer lies in graph theory. If we represent each island as a vertex and each bridge as an edge, then the seven bridges form an undirected graph. Now, an undirected graph has an Eulerian circuit iff there is a path through the graph that crosses every edge exactly once. The graph corresponding to the seven bridges lacks an Eulerian circuit and so there is no such path. It follows that there is no way of crossing the seven bridges, crossing each bridge exactly once. This gives us the following explanation:

Bridge: There is no way of crossing the seven bridges of Königsberg passing over each bridge exactly once because the bridges have the structure of a graph that lacks an Eulerian circuit.

Here is a nearby purely mathematical case. Let a net for an $n$-dimensional figure be a continuous $n-1$ dimensional figure that can be folded into the $n$-dimensional figure without being overlaid back onto itself. So, for example, a net for a three-dimensional figure like a cube is a continuous two-dimensional figure like a Latin cross, that can be folded into the cube without overlaying any part of the cross onto itself. It is an outstanding problem in mathematics to prove that every concave polyhedron has a twodimensional net in this sense (this is known as Shepherd's conjecture). Focus, however, on the case of a two-dimensional figure, like a triangle. A triangle has a one-dimensional net. The one-dimensional net is just a line (all one-dimensional nets are). Not every two-dimensional figure has a net, however. Consider a square with a cross through the middle, such as the figure on the left in Figure 1. There is no continuous one-dimensional line that can be folded into that figure (we could do it if we let the line overlay back onto itself, but that wouldn't be a net).
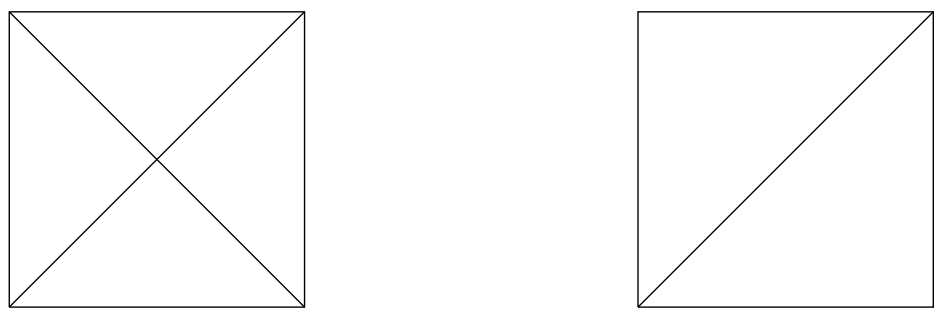
Figure 1: The left-hand figure has a one-dimensional net, the right-hand figure does not.

Why does the left-hand figure in Figure 1 lack a net? Well, suppose we model the left-hand figure as a graph. Then a net for the left-hand figure is a line that can be folded to produce the figure without overlapping, and such a line would correspond to a path through the graph that crosses over every edge exactly once. Thus, Figure 1 has a net only if the corresponding graph possesses an Eulerian circuit. The graph corresponding to Figure 1 lacks an Eulerian circuit, however, and so there is no net. Contrast this with a triangle. A triangle does correspond to a graph with an Eulerian circuit, and so there is a one-dimensional net for that figure. Or consider a square with a single diagonal line (on the right in Figure 1). This figure also corresponds to a graph with an Eulerian circuit, and so there exists a one-dimensional net.

Corresponding to Bridge, then, we have:

Net: There is no one-dimensional net corresponding to the left-hand figure in Figure 1 because that figure has the structure of a graph that lacks an Eulerian circuit.

We can then combine Bridge and Net to get a unified explanation of both:

Bridge-Shape: There is no way of crossing the seven bridges of Königsberg passing over each bridge exactly once and there is no one-dimensional net corresponding to the left-hand figure in Figure 1 because both the bridges and the figure have the structure of a graph that lacks an Eulerian circuit.

Yarn-Trefoil and Bridge-Shape provide a preliminary sense of the kind of case needed to support the argument offered in $\S 2$. What we need, though, are realistic scientific cases. Realistic examples are harder to come by, however. That's because realistic $R$-explanations typically use a range of physical features to explain a given physical explanandum. As such, any mathematical fact that is outputted by such an explanation would seem to admit of a partly physical explanation after all, something that I have ruled out. It is thus difficult to see how a single realistic $R$-explanation could unify both a mathematical and a physical fact. In the next section, I will refocus the argument outlined in $\$ 2$ so that it is more readily applicable to scientific examples. This will enable me to then identify one potential source of realistic cases

\section{A Potential Source of Scientific Cases}

The type of vertical unification discussed thus far involves a single $R$-explanation that yields both a physical and a mathematical fact as outputs. There is, however, another kind of vertical unification often found within science. Rather than having a single $R$ explanation that yields two distinct explananda, we might have two very similar but distinct $R$ explanations that yield $A$ and $B$ respectively and that are produced from a more general 'recipe' or 'pattern' which is itself a part of some theory. In this case we may say that $A$ and $B$ enjoy indirect vertical unification in virtue of the fact that they have distinct $R$ explanations that are both instances of the same general pattern.

The appeal to patterns of explanation is reminiscent of Kitcher's (1981) account of scientific explanation. Kitcher takes $R$-explanations to be arguments. A particular argument 
counts as an $R$-explanation if it is an instance of an argument pattern that itself is a member of a set of patterns that provides the best way of unifying our empirical beliefs overall. Kitcher's view, however, has been widely criticised, and I am not advocating it here. The point is just that whatever flaws Kitcher's account might have, it surely gets something right about science. There are clearly patterns within our best scientific theories that provide a recipe for producing specific $R$-explanations. Indeed, it is something of a goal of science to produce broad patterns of explanation that can be applied across a range of cases.

A pattern, I will assume, is just a set of sentences, some of which have been generalised by replacing specific details with variables, such that a specified range of values can be substituted back in for the variables in question (or a similarly generalised model). An $R$-explanation $E$ is an instance of a pattern $\Sigma$ just when there is a uniform substitution of variables in $\Sigma$ that produces $E$. Here's a rough example. Suppose that Sara and Suzy each strike a match and the match lights. This gives us two explananda: (i) Sara's match lit and (ii) Suzy's match lit. The two explananda have distinct $R$-explanations: one $R$ explanation appeals to Sara's striking; the other $R$-explanation appeals to Suzy's striking. Despite being distinct, both $R$-explanations can be generated from a common pattern of explanation, which can be stated in general argument form as follows:

1. For any match $M$, if $M$ is struck under conditions $C_{1} \ldots C_{n}$, then $M$ will light.

2. $M$ is struck under conditions $C_{1} \ldots C_{n}$. So,

3. $M$ will light.

To get the two match-lighting $R$-explanations, we simply substitute in the details of Suzy's striking or Sara's striking. Both $R$-explanations make use of a single general fact, namely: the fact that for any match $M$, if $M$ is struck under conditions $C_{1} \ldots C_{n}$, then $M$ will light.

Unification under a general pattern is not always desirable. As Kitcher (1981) showed, there can be cases of spurious unification. Unification under a general pattern is spurious when the explanations that are unified by the pattern, should not be unified. It is notoriously difficult to give an account of the difference between spurious and non-spurious unification for patterns. However, one plausible constraint on non-spurious unification relates to the distinction between $R$-explanations and $O$-explanations discussed earlier. The constraint is this: two $R$-explanations are non-spuriously unified under a common pattern only if the $O$-explanations represented by the $R$-explanations at issue share some common feature. Note that the $O$-explanations need not be identical; there just needs to be some aspect of the $O$-explanations that is held in common. If the $O$-explanations for two facts share nothing in common, it is a mistake to unify the $R$-explanations of those facts at any level within science.

Again, scientific realism is an important element of the motivation for the proposed constraint on non-spurious unification. When our scientific theories are true, they accurately track $O$-explanations. When we unify two distinct $R$-explanations under a common pattern, we thereby represent their corresponding $O$-explanations as having some commonality between them; a commonality captured by the pattern in question. If our theories represent two $O$-explanations as sharing a common feature, and those $O$-explanations share nothing in common, our theories seem to misrepresent the world. Partly what unification 
under a general pattern is doing within our scientific theories is providing information about where explanatory commonalities lie.

Using the notion of unification under a pattern, we can formulate an argument that parallels the one given in $\S 2$ :

(1) A physical explanandum $P$ and a mathematical explanandum $M$ are vertically unified under a common pattern $\Sigma$.

(2) If $P$ and $M$ are vertically unified under a common pattern $\Sigma$, then their $O$-explanations share some common feature.

(3) The $O$-explanation for every mathematical fact is mathematical.

(4) If (1), (2) and (3), then the common feature between the $O$-explanations for $M$ and $P$ is mathematical in nature.

(5) If the common feature between the $O$-explanations for $M$ and $P$ is mathematical in nature, then there is at least one physical fact $P$ that obtains, in part, for mathematical reasons.

Therefore,

(6) There is at least one physical fact $P$ that obtains, in part, for mathematical reasons.

As before, Premise (1) is an assumption. Premise (2) follows from the constraint on nonspurious unification just discussed. Premise (3) was defended in §2. Given Premise (3), the shared feature between the $O$-explanations for $P$ and $M$ must be mathematical in nature, because there are no non-mathematical features of the $O$-explanation for $M$. From this it follows, as per Premise (5), that there are physical explananda that hold, in part, for mathematical reasons.

If we focus on indirect vertical unification, it becomes easier to see how there could be realistic scientific examples in which physical and mathematical explananda are explanatorily unified. As noted, most $R$-explanations of physical phenomena appeal to physical facts and so it is difficult to see how the very same $R$-explanations might explain mathematical facts as well. There is, however, no general requirement for two $R$-explanations that fall under a single pattern to feature the very same facts. So an $R$-explanation that explains a mathematical fact need not feature any physical facts, despite being vertically unified with an $R$-explanation that does.

Of course, some patterns of explanation will impute a requirement on their instances to feature certain physical facts. The match-lighting case described above is like this: every instance of that pattern must refer to a physical object - a match — and a physical law that connects match-lightings to match-strikings. But whether a pattern of explanation imputes a requirement along these lines depends very much on the pattern in question. In particular, whether a pattern of explanation forces all of its instances to feature physical facts plausibly depends on how general the relevant pattern is. This can be seen by considering the degenerate case: a pattern that is so general that it applies to any physical system whatsoever. Such a pattern cannot require of its instances that they feature any particular physical facts or else it will rule out some physical case or other. Realistic patterns won't be as general as the degenerate pattern, but some may still be so general that they fail to specify any strict physical requirements in a similar way. 
The kind of generality being hinted at is what Baker (2017) calls topic generality. Roughly put, the topic generality of a particular pattern relates to how much the pattern abstracts away from specific physical detail. As we increase the topic generality of a pattern, we typically weaken the requirement that the pattern imposes on its instances to feature any specific physical facts. This, in turn, opens the way for the pattern to have a pure mathematical instance. In this way patterns of explanation with a high level of topic generality have the potential to indirectly unify physical and mathematical explananda.

By focusing on topic generality we can thus gain some insight into where we might find patterns of explanation that support the argument outlined in $§ 2$. Obviously, we should be looking at patterns of explanation that abstract away from physical detail as much as possible. More than this, however, we should be looking at patterns of explanation that indirectly unify quite disparate physical explananda. Indeed, the best kind of case to consider is one where the $R$-explanations at issue apply to such diverse phenomena that they make no common appeal to a particular physical fact or class of physical facts. A pattern that manages to subsume the $R$-explanations at issue cannot reasonably impose a requirement on its instances to specify any physical feature in particular.

In recent work, Baker (2017) presents two $R$-explanations for two very different physical explananda and suggests that they should be unified under a common pattern. A pattern capable of unifying the two cases he identifies (or cases like them) does seem to have a pure mathematical instance as well. In what follows I will briefly sketch out Baker's case. I should note, however, that I am not offering the case as a conclusive example of a scientific explanation that supports the argument offered in this section. Rather, I offer the case as a way of demonstrating how topic generality might result in the indirect unification of physical and mathematical explananda, and as a prototype for finding more realistic scientific case studies in the future. Even if what I say about the case is wrong, the general point made in this section stands, namely that we should be looking for cases in which disparate physical phenomena are unified, since such cases may require explanations so general that they reach all the way into mathematics.

With this in mind, let us turn to the case itself. The case centres around Baker's wellknown example of the prime-numbered life-cycle lengths of North American cicadas. The North American magicicada spends most of its life in the ground in its larval state. When conditions are right, it arises in a grand swarm as an adult for around two weeks during which it eats, breeds, dies and repeats the cycle. There are two sub-species of the magicicada that possess life-cycles of 13 and 17 years respectively. Why these life-cycles in particular?

Well, assume, first, that there are ecological constraints that force the cicadas to have a life-cycle in the 12-18-year range. Next, assume that, in the past, the magicicada was predated by a range of predators with life-cycles of 2, 3, 4, 5, 6, 7, 8 and 9 years. The optimal strategy for avoiding predation in this scenario is to minimise the frequency of intersection between magicicada swarming and predator swarming. Having a prime-numbered lifecycle achieves this result. For any two numbers $m$ and $n$ their lowest common multiple is maximised when $m$ and $n$ are coprime. 13 and 17 are both coprime with each of 2, 3, 4, 6, 7, 8 and 9. Accordingly, the lowest common multiple between 13 or 17 and numbers in the 2-9 range is maximised, compared to other numbers in the $12-18$ range. Because the frequency of intersection between the life-cycles of the magicicada and any predators is lowest when the lowest common multiple of the life-cycles is maximised, it follows that the optimal life-cycle for predator avoidance in the 12-18-year range is 13 or 17. Cicadas with 
periodical life-cycles that are being predated by predators that have periodical life-cycles should evolve toward this optimum. ${ }^{14}$

The cicada case can be unified with another example that has nothing to do with biological organisms. The second case focuses, instead, on gear ratios within mechanical systems. Baker (2017) focuses on gear-ratios in fixed-speed bicycles, but, as Baron (2020) discusses, a similar kind of case arises for any mechanical system in which large gears turn small gears to produce a mechanical advantage. It is the more general kind of case that I will focus on here.

Suppose we aim to build a mechanical system featuring large gears turning small gears that minimises wear and tear on the gears in question. Gears are typically produced with a small bump on at least one of their teeth as a byproduct of the manufacturing process. If the bump on a large gear repeatedly strikes the same spot on a small gear, then the tooth on the small gear will wear down, and will eventually break, leading to mechanical failure. It is imperative, then, to minimise the frequency with which the bump on a large gear strikes the same spot on a small gear.

Suppose further that we have a range of small gears to choose from, each of which has between 2 and 9 teeth and we have a choice of large gears that have between 12 and 18 teeth. Which large gears should we choose to turn our small gears so as to minimise wear and tear on the system? The answer is that we should use gears with 13 or 17 teeth. The reason for this has to do with the relationship between the rotational periods of large and small gears. In one unit of the gear's rotation period every tooth on the gear moves through an angle $m^{\circ}$ where $m=\frac{360}{n}$ and $n$ is the number of teeth. So, for instance, if a gear has 9 teeth, then one unit of the gear's period is a rotation of every tooth through $40^{\circ}$. It will take 9 units of rotation to return the teeth back to their original position. The gear's rotation period is thus equivalent to its number of teeth.

Now, we want to ensure that the bump on a particular tooth on a large gear-say tooth 2 - doesn't keep striking a particular tooth on a small gear-say tooth 3 . If the number of teeth on the large gear is co-prime with the number of teeth on the small gear, then the rotation period of the large gear will be co-prime with the rotation period of the small gear. This, in turn, means that the number of full gear rotations between any pair of teeth striking one another will be maximised, which will ensure that the small gear lasts as long as possible. Given the options, then, large gears with 13 or 17 teeth minimise wear and tear on small gears because any pair of teeth consisting of a tooth on the large gear and a tooth on the small gear will come into contact much less frequently than for any other large gear size within the specified range. This is for the same reason that 13 and 17-year life-cycles maximise predator avoidance. The frequency with which a single tooth on the large gear hits the same tooth on the small gear is a function of the lowest common multiple of the rotational periods of the two gears.

Thus, the gear case and the cicada case use the same underlying mathematics. They do not, however, seem to share anything physical in common. The two systems involve different physical entities (cicadas versus gears), are governed by different physical laws (laws of evolution and natural selection versus mechanical laws) and involve different kinds of periodical phenomena (life-cycle lengths versus gear rotations measured in teeth). Indeed, there appear to be just three commonalities between the two cases: (i) the two cases feature the same mathematics; (ii) in both cases there are various phenomena that cycle or repeat

\footnotetext{
${ }^{14}$ See Baker (2009); Baker and Colyvan (2011); Baker (2017) for defence of the cicada case.
} 
and (iii) in both cases there is a kind of 'pressure' to maximise something.

A pattern that manages to unify the two $R$-explanations at issue, then, should really only require its instances to feature these three general aspects. If the pattern is any more stringent, it will likely rule out one of the two cases. Thus, to capture (ii) the pattern should feature a general way of referring to repeating phenomena that abstracts away from the specific physical features of cicada life-cycle lengths and gear rotations. Similarly, to capture (iii) the pattern should feature a general way of referring to a feature to be maximised or minimised along with a general way of specifying the pressure to maximise or minimise this feature, again without including any of the specific physical aspects of the cicada or gear cases. A general statement of (i) will feature a broad mathematical framework that can be applied to the two cases equally.

A pattern possessing just these three features, specified at such a general level, is likely to have a mathematical instance. To see this, let us start with the first general feature of the pattern: the mathematical framework. Baker formulates a general mathematical framework for the two cases in terms of unit-cycles. A cycle is a mathematical sequence in which the elements of the sequence are repeated in a specified order. The period of the sequence corresponds to the number of repeated elements that are members of the sequence. The period is also how many 'steps' it takes the cycle to return to the point at which it began. So, for example, the following sequence of numbers: $1,2,3,1,2,3$ is a cycle, the period of which is 3 .

A unit cycle is a repeating sequence that is part of a larger structure, and is such that the period of the cycle is always a multiple of a fixed base unit. In the cicada case, we can take the larger structure to be time, the fixed base unit to be a year, and the unit cycles to be repeating patterns of $n$ years. The cicada life-cycle can thus be modelled as a unit cycle of the numbers $1,2,3,4,5,6,7,8,9,10,11,12,13$ and the life-cycles of the cicada predators can be modelled as shorter cycles such as: 1, 2 (two year predators), 1, 2, 3 (three year predators), 1, 2, 3, 4 (four year predators) and so on. In the gear case, we can take the larger structure to be gear rotations, the fixed base unit to be one tooth, and the unit cycles to be repeating patterns measured in teeth (i.e., the number of teeth it takes the gear to return to its starting point).

Having modelled the cicada and gear cases using unit-cycles, Baker then proposes the following as the mathematical core of the two explanations introduced above:

1. The lowest common multiple (LCM) of two numbers $m, n$ is maximal if and only if $m$ and $n$ are coprime.

2. The gap between successive co-occurences of the same pair of cycle elements of two unit cycles is equal to the LCM of their respective lengths.

3. If [1] and [2] then any pair of unit cycles with periods $m$ and $n$ maximises the gap between successive co-occurrences of the same pair of cycle elements if and only of $m$ and $n$ are coprime.

4. All and only prime numbers are coprime with all smaller numbers.

5. If [3] and [4] then given a unit cycle, $p_{m}$, of length $m$ and a range of unit cycles, $q_{i}$, of lengths shorter than $m, p_{m}$ maximises the gap between successive co-occurrences of the same pair of cycle elements with each $q_{i}$ if and only if $m$ is prime. 
Using this mathematical core we can derive the fact that cicadas should have primenumbered life cycles as follows. Whenever there is a co-occurrence of cycle elements between the unit cycle that corresponds to the cicada life-cycle and the unit cycle of any predator life-cycle, the predators and the cicadas emerge from the ground together. Thus predator avoidance is a matter of maximising the gap between the successive co-occurrence of cycle elements. Since having a life-cycle that corresponds to a unit cycle $u$ of length $m$ where $m$ is a prime number maximises this gap, and since the length of a unit-cycle just is the length of a life-cycle in years, the cicadas should evolve to have a prime-numbered life-cycle in order to maximise predator avoidance.

The gear case can be derived in basically the same way. The smallest wear and tear on gears corresponds to the largest gap between the successive co-occurrence of cycleelements for unit-cycles that represent gear rotations. A prime-numbered rotation period for a large gear thus minimises the wear and tear of that gear on all smaller gears, and so large gears should have a prime numbered amount of teeth, because the length of a unit-cycle that corresponds to gear rotations just is the number of teeth possessed by the gear.

We can also use the mathematical core to derive mathematical facts about sequences. Here's a rough example. Suppose we have a set $Q$ of ordered, repeating sequences $S_{1} \ldots S_{n}$, each with different periods. And suppose we have, in addition, two further sequences $S$ and $S *$ with periods $r$ and $r *$ respectively that are not in the set and where $r$ is prime and $r *$ is not. Finally, suppose that the periods for the $S_{n}, r_{1} \ldots r_{n}$ are such that $r_{n}<r$ and $r_{n}<r *$. Now, suppose we discover by a process of manually counting elements, that the gaps between the successive co-occurrences of elements in $S_{1}$ and each member of $Q$ are greater than the gaps between the successive co-occurrences of elements in $S_{2}$ and each member of $Q$. Why should this be the case?

We can answer this question by noting that each sequence is a unit-cycle, where the base unit is simply 1 and the larger structure is just the natural numbers. We can thus apply the mathematical core that Baker sketches in the same way we might for the gears or for the cicadas. When we apply the core, we discover that the gaps between successive cooccurrences of elements in $S_{1}$ and each member of $Q$ is greater than between $S_{2}$ and each member of $Q$. This is because only $r$ is prime and thus only $r$ is co-prime with each $r_{i}$.

Now, this mathematical example is not yet an instance of the same pattern of explanation that captures the cicada and gear cases. The example satisfies the first general feature of the pattern, since it can be modelled via the mathematical core, and it satisfies the second general feature because it involves repeating phenomena. However, what we are missing is something that satisfies the third general feature of the pattern, namely a pressure to maximise something, and a value for whatever is being maximised. It is not difficult to set up the case, however, so that it satisfies this aspect of the pattern as well.

We start, as before, with a set $Q$ of ordered, repeating sequences $S_{1} \ldots S_{n}$, each with periods $r_{1} \ldots r_{n}$. This time, however, suppose we have another set $P$, which features a number of sequences $S *_{1} \ldots S *_{n}$ with periods $r *_{1} \ldots r *_{n}$ such that (i) for each $r_{i}$ and for each $r *_{i}, r_{i}<r *_{i}$ and (ii) the difference between any $r *_{i}$ and any $r_{i}$ is no greater than 10 . Now, suppose we introduce a function over $P$ call it $f$ that selects only those sequences in $P$ that have the largest gap between successive co-occurrences of their elements and the elements of each sequence in $Q$ and maps them to $1 \in\{1,0\}$. All other sequences in $P$ are mapped to $0 \in\{1,0\}$. We discover that this function maps only those sequences in $P$ that have prime-periods to 1 . Why? The reasoning should, I hope, be obvious: the 
sequences at issue are unit-cycles. The function $f$ should thus map only those sequences with prime periods to $1 \in\{1,0\}$ because only those sequences will have periods that are co-prime with every period of every sequence in $Q$ and the distance between successive co-occurrences of elements for any two sequences is largest iff their periods are co-prime.

This explanation of why $f$ picks only prime members of $P$ appeals to the fact that $f$ 'aims' to maximise some value. The value itself is just the distance between the successive co-occurrence of elements between unit cycles that are mapped by $f$. This is nonetheless analogous to the way in which the explanation for why cicadas have prime-numbered lifecycles appeals to the fact that the cicadas 'aim' to maximise fitness, or the way in which machine manufacturers aim to minimise wear and tear on small gears. Indeed, a mathematical case along these lines just is a pure mathematical analogue of the cicada and gear cases. In this way it is very similar to the toy cases outlined in the previous section, each of which involves finding a pure mathematical analogue of a certain distinctively mathematical explanation of a physical phenomenon. The difference being, however, that in the present context, the mathematical analogue is unified under a general explanatory pattern, rather than being unified under a single $R$-explanation. ${ }^{15}$

As noted, I offer Baker's case only as a prototype for finding a realistic case study that supports the argument offered in $\S 2$. The trouble with the case is that no scientist to date has sought to unify the cicada and gear cases together under a general pattern of the kind described here, let alone under a general pattern that has a pure mathematical instance. This is why I have not formulated a general pattern that covers the cicada, gear and mathematical cases in complete detail. And why I have only really gestured, in broad terms, at how such a pattern might be formulated, and why it may have a mathematical instance. For even if I formulate a general pattern that unifies the three cases together, the pattern itself is still open to the charge of artificiality.

This issue with Baker's case not withstanding, the case is still a useful template for identifying realistic scientific examples. Moreover, there is reason to be optimistic that there are examples of the right kind to be found. For it is notable that science-especially physics - has come to focus more and more on the provision of very general explanations that ignore a great deal of physical detail. The more topic general our explanations become, and the more they ignore physical features of the world in favour of mathematical ones, the more likely it is that physical phenomena and mathematical phenomena will be explained together. If I am right, then finding such a case promises a significant pay-off, for it would show that mathematics is playing a thick role in scientific explanation after all.

Now, some may not be quite so optimistic about finding cases of the right kind. In recent work, Knowles and Saatsi (2019) argue that when we unify two disparate explananda under a common pattern, we don't gain a deeper understanding of the explananda at issue. Such unification provides, at best, a way to formulate explanations in a manner that is cognitively salient. It is therefore unlikely that our best scientific theories will feature unification of the relevant kind, since it adds nothing of substance when it comes to expla-

\footnotetext{
${ }^{15}$ One might worry about the example: haven't I just shown that there is a pure mathematical explanation of a mathematical fact embedded within the cicada and gear cases? And doesn't this just make the case into a variant on one of Steiner's, which I have already said provide no support for the argument in $\$ 2$ ? Note, however, that there is no reason why the sequences in the mathematical case have to be the same sequences as in the cicada or gear cases. Assume that they are different sequences. Then the mathematical explanation is still an instance of the general pattern and it is not embedded in the cicada and gear cases, because those cases involve entirely different sequences.
} 
nation.

The argument offered by Knowles and Saatsi deserves greater attention than I can offer it here. I will, however, close by offering two points in response. First, Knowles and Saatsi argue that if unification yields no deeper understanding of the explananda unified, then it provides no explanatory benefits to our theories. This, however, is not so obvious. According to Kitcher (1981, pp. 529-530), one of the chief contributions of unification is to reduce the number of mysteries that there are in the world. By unifying explananda under a small stock of common patterns, we thereby reduce the number of phenomena we need to take as brute or unexplained. Thus, while unification may not yield further understanding of specific explananda, it may nonetheless contribute to explanatory power in another sense.

Second, and relatedly, even if Knowles and Saatsi are right that unification yields no explanatory benefits, it may nonetheless yield benefits of a different kind; benefits that ultimately factor into theory choice when selecting between theories. Indeed, it is possible to see unification as a theoretical virtue independently of its connection to explanation (cf. Myrvold (2017)). Accordingly, scepticism about the link between unification and explanation need not translate into scepticism about the link between unification and truth. We may still find, then, that there is pressure for our best scientific theories to include unification of the kind discussed here. So long as there is some drive toward this type of unification, there is a basis for cautious optimism regarding the unification of physical and mathematical phenomena within science.

Acknowledgements I would like to thank audience members at the University of Lisbon for helpful discussion of an earlier version of this paper, particularly Amanda Bryant and David Yates. Research on this paper was supported by a Discovery Early Career Researcher Award from the Australian Research Council, DE180100414.

\section{REFERENCES}

Andersen, Holly (2016), "Complements, not competitors: Causal and mathematical explanations.” British Journal for the Philosophy of Science, 69, 484-508.

Baker, Alan (2005), “Are there genuine mathematical explanations of physical phenomena?" Mind, 114, $223-238$.

Baker, Alan (2009), "Mathematical explanation in science." British Journal for the Philosophy of Science, $60,611-633$.

Baker, Alan (2012), "Science-driven mathematical explanation.” Mind, 121, 243-267.

Baker, Alan (2017), "Mathematics and explanatory generality.” Philosophia Mathematica, 25, 194-209.

Baker, Alan and Mark Colyvan (2011), "Indexing and mathematical explanation." Philosophia Mathematica, $19,323-334$.

Bangu, Sorin (2020), "Mathematical Explanations of Physical Phenomena." Australasian Journal of Philosophy, https://doi.org/10.1080/00048402.2020.1822895

Baron, Sam (2020), “Counterfactual scheming.” Mind, 129, 535-562.

Baron, Sam, Mark Colyvan, and David Ripley (2017), "How mathematics can make a difference." Philosophers' Imprint, 17. 
Bueno, Otávio (2012), “An easy road to nominalism.” Mind, 121, 967-982.

Chirimuuta, Mazviita (2018), "Explanation in Computational Neuroscience: Causal and Non-Causal." British Journal for the Philosophy of Science, 69(3), 849-880.

Colyvan, Mark (2001), The Indispensability of Mathematics. Oxford University Press, Oxford.

Colyvan, Mark (2002), "Mathematics and aesthetic considerations in science.” Mind, 111, 69-74.

Colyvan, Mark (2010), “There is no easy road to nominalism.” Mind, 119, 285-306.

Field, Hartry (1980), Science Without Numbers. Oxford University Press, Oxford.

Frost-Arnold, Greg (2010), “The no-miracles argument for realism: Inference to an unacceptable explanation." Philosophy of Science, 77, 33-58.

Jansson, Lina and Juha Saatsi (2019), "Explanatory abstractions." British Journal for the Philosophy of Science, 70, 817-844.

Kitcher, Philip (1981), “Explanatory unification.” Philosophy of Science, 48, 507-531.

Kitcher, Philip (1984), The Nature of Mathematical Knowledge. Oxford University Press, Oxford.

Knowles, Robert (forthcoming), "Platonic relations and mathematical explanations.” Philosophical Quarterly.

Knowles, Robert and Juha Saatsi (2019), “Mathematics and explanatory generality: Nothing but cognitive salience.” Erkenntnis, https://doi.org/10.1007/s10670-019-00146-x.

Lange, Marc (2016), Because Without Cause. Oxford University Press.

Leng, Mary (2010), Mathematics and Reality. Oxford University Press, Oxford.

Liggins, David (2012), "Weaseling and the content of science.” Mind, 121, 997-1005.

Lyon, Aidan (2012), "Mathematical explanations of empirical facts, and mathematical realism." Australasian Journal of Philosophy, 90, 559-578.

Melia, Joseph (2000), "Weaseling away the indispensability argument.” Mind, 109, 455-479.

Myrvold, Wayne C. (2017), “On the evidential import of unification.” Philosophy of Science, 84, 92-114.

Pincock, Christopher (2007), “A role for mathematics in the physical sciences.” Nô̂s, 41, $253-275$.

Pincock, Christopher (2015), “Abstract Explanations in Science.” British Journal for the Philosophy of Science, 66(4), 857-882.

Potochnik, Angela (2007), “Optimality modeling and explanatory generality.” Philosophy of Science, 74, 680-691.

Povich, Mark (2019), “The Narrow Ontic Counterfactual Account of Distinctively Mathematical Explanation.” British Journal for the Philosophy of Science, https://doi.org/10.1093/bjps/axz008.

Psillos, Stathis (1999), Scientific Realism: How Science Tracks Truth. Routledge, London.

Saatsi, Juha (2011), "The enhanced indispensability argument: representational vs. explanatory role of mathematics in science." British Journal for the Philosophy of Science, 62, 143-154.

Saatsi, Juha (2016), "On the "indispensable explanatory role” of mathematics.” Mind, 125, 1045-1070. 


\section{REFERENCES}

Saatsi, Juha (2017), "Dynamical systems theory and explanatory indispensability." Philosophy of Science, 84, 892-904.

Saatsi, Juha T. (2007), "Living in harmony: Nominalism and the explanationist argument for realism." International Studies in the Philosophy of Science, 21, 19-33.

Skow, Bradford (2015), “Are there genuine physical explanations of mathematical phenomena?" British Journal for the Philosophy of Science, 66, 69-93.

Steiner, Mark (1978a), "Mathematical explanation.” Philosophical Studies, 34, 131-151.

Steiner, Mark (1978b), "Mathematics, explanation, and scientific knowledge.” Nô̂s, 12, 17-28.

Yablo, Stephen (2012), “Explanation, extrapolation and existence.” Mind, 121, 1007-1029.

Zelcer, Mark (2013), “Against mathematical explanation.” Journal for General Philosophy of Science, 44, 173-192. 